\title{
Breaking the curse of dimensionality to identify causal variants in Breeding 4
}

\author{
Guillaume P. Ramstein ${ }^{1}$ (D) Sarah E. Jensen ${ }^{2}$ (i) $\cdot$ Edward S. Buckler ${ }^{1,2,3}$ (])
}

Received: 15 November 2018 / Accepted: 7 December 2018 / Published online: 13 December 2018

(c) The Author(s) 2018

\begin{abstract}
In the past, plant breeding has undergone three major transformations and is currently transitioning to a new technological phase, Breeding 4. This phase is characterized by the development of methods for biological design of plant varieties, including transformation and gene editing techniques directed toward causal loci. The application of such technologies will require to reliably estimate the effect of loci in plant genomes by avoiding the situation where the number of loci assayed $(p)$ surpasses the number of plant genotypes $(n)$. Here, we discuss approaches to avoid this curse of dimensionality $(n \ll p)$, which will involve analyzing intermediate phenotypes such as molecular traits and component traits related to plant morphology or physiology. Because these approaches will rely on novel data types such as DNA sequences and high-throughput phenotyping images, Breeding 4 will call for analyses that are complementary to traditional quantitative genetic studies, being based on machine learning techniques which make efficient use of sequence and image data. In this article, we will present some of these techniques and their application for prioritizing causal loci and developing improved varieties in Breeding 4 .
\end{abstract}

\section{Past and current trends in plant breeding}

This perspective article considers technologies that have contributed to the evolution of plant breeding and focuses on innovations that will shape its future, amid a growing world population and changing climate. Since the Neolithic revolution, plant breeding has gone through distinct phases, separated by radical transformations. Within each of these major technological phases, we see a pattern that we would now call a shift from monogenic to polygenic focus. While it is tempting to believe this pattern is the product of greater understanding, it is most likely the product of how adaptation proceeds, as suggested by the Fisher-Orr geometric model (Fisher 1930; Orr 1998). A population evolving

Communicated by Rod Snowdon.

Guillaume P. Ramstein

gr226@cornell.edu

1 Institute for Genomic Diversity, Institute of Biotechnology, Cornell University, 175 Biotechnology Building, Ithaca, NY 14853, USA

2 Section of Plant Breeding and Genetics, Cornell University, Ithaca, NY 14853, USA

3 United States Department of Agriculture, Agricultural Research Service, Ithaca, NY 14853, USA toward a new evolutionary optimum is more likely to substitute large-effect mutations in the first generations, while subsequent generations continue to fix smaller-effect mutations. Over time, it generally takes orders of magnitude more small effects to climb the population's adaptive peak (Orr 2005). So, as new evolutionary optima have been set by environmental changes (e.g., domestication, management practices, biotic or abiotic pressures) or major shifts in genetic background, plant breeders have selected better-adapted genotypes while pursuing faster genetic gains through technological innovations and scientific breakthroughs. As these discoveries marked transformations in plant breeding, different phases have built upon one another, each time expanding the set of tools available for making progress (Fig. 1).

The first phase, which we refer to here as Breeding 1, accounts for the first 10,000 years of crop improvement. Early plant breeders domesticated useful species and unintentionally promoted domestication traits, such as reduced shattering in maize (Stitzer and Ross-Ibarra 2018) and grain composition in rice (Kovach et al. 2007) (Breeding 1.1). These domestication traits likely consisted of a few major genes controlling dramatic changes in crop physiology, as is suggested by genetic studies comparing maize to its ancestor teosinte (Doebley et al. 1990; Briggs et al. 2007). Early domestication phases were followed by long periods of deliberate selection for increased performance, probably 


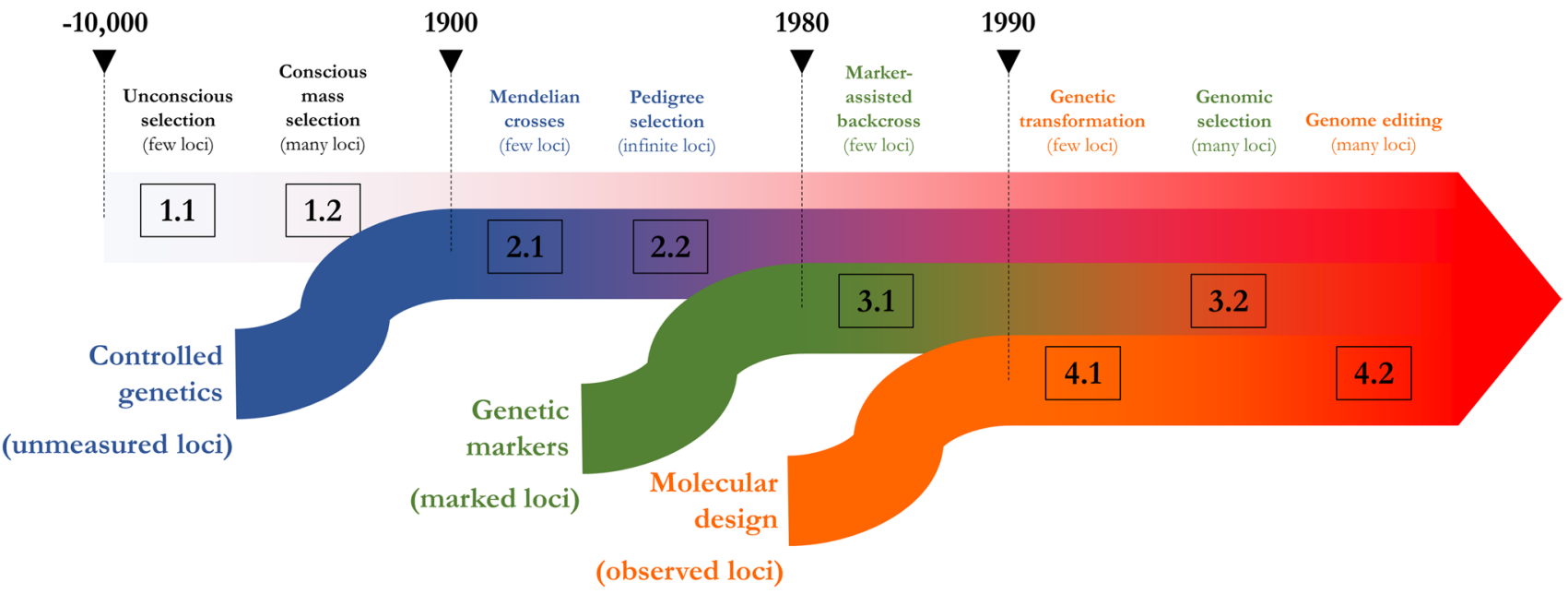

Fig. 1 Timeline of plant breeding phases. Breeding 1, selection with unknown loci; Breeding 2, selection by controlled crosses; Breeding 3, marker-assisted selection; Breeding 4, ideotype-based selection and transformation

involving mass selection for traits with a complex polygenic basis, such as plant environmental adaptations and yield (Breeding 1.2).

The second major phase in plant breeding, Breeding 2, began much later, in the early- and mid-twentieth century, and was characterized by the understanding and implementation of Mendelian genetics (Mendel 1866; De Vries 1900; Correns 1900) as well as the development of methods to account for environmental variability by experimental design (Fisher 1935; Cochran and Cox 1950). These advances allowed introgression of important genes for modern agriculture (Biffen 1905), including disease resistance genes under the gene-for-gene hypothesis (Flor 1971) and dwarfing genes in wheat and rice which enabled the Green Revolution (Hedden 2003) (Breeding 2.1). This phase was also marked by the development of familial crosses brought about by critical innovations in statistical science (Breeding 2.2). The genetic theory of resemblance between relatives initiated by Fisher (1919) and Wright (1921) supported impactful breeding schemes (e.g., sib mating and selfing) which resulted in higher selection gains by increased heritability at the family level (among-family selection being more accurate than mass selection) and hybrid vigor by inbred crosses (making use of genomes' complementarity) (Bernardo 2002). Furthermore, a theoretical framework to predict genetic gains was introduced by the "breeder's equation," attributed to Lush (1937) (Turelli 2017). Finally, linear mixed models developed by Henderson et al. (1959) provided a valuable tool to account for familial relationships in the prediction of genetic merit, initially in animal breeding and, much later, in plant breeding (Panter and Allen 1995).

The third phase in crop improvement, Breeding 3, added genomics to the breeder's toolbox. Marker-assisted breeding made it possible to consider both phenotype data on agronomic traits and genotype data from DNA information. Effects of polymorphisms could finally be estimated, and causal loci previously unmeasured in Breeding 2 became exposed, though indirectly, by association between phenotypes and genetic markers physically linked to causal loci. The first breeding applications of genomics consisted in mapping quantitative trait loci (QTL) in experimental populations, using genetic markers such as restriction fragment length polymorphisms (Soller et al. 1976; Stuber et al. 1980; Tanksley et al. 1982). Applications of such inference techniques were marker-assisted backcross selection schemes (Hospital et al. 1992), which allowed faster and more precise introgression of major QTL for phenotypes like disease resistance and morphological traits (Breeding 3.1). QTL models, initially designed for analyzing few genetic loci, were then extended to genome-wide models incorporating information about many genetic markers across the genome, such as genomic prediction models (Meuwissen et al. 2001). In major crops like maize and rice, using genomic prediction approaches has become common practice to compare and select the best individuals for complex traits (Heslot et al. 2015; Bevan et al. 2017), while genome-wide association mapping has successfully identified QTL for relatively simple traits related to flowering and leaf architecture (Huang and Han 2014) (Breeding 3.2). As genotyping and phenotyping technologies become cheaper, breeders will likely expand these techniques to new crops and in smaller breeding programs. Even though Breeding 3 began nearly 30 years ago, the use of genomics in crop improvement is far from widespread across species and regions of the globe. Future plant breeding will expand Breeding 3 globally and will begin the transition to Breeding 4 (Fig. 1). 


\section{Statistical limitations in plant breeding and the transition to Breeding 4}

\section{The concept of causal loci and the curse of dimensionality}

The concept of loci controlling agronomic traits has been at the core of breeders' ability to describe and exploit genetic variability. As breeding science progressed, a better understanding of causal loci has increased breeders' control over the genetics of complex traits. In Breeding 1, the basis for variability at agronomic traits was unknown, making mass selection the only viable breeding scheme. In Breeding 2, the concept of a causal locus ("Mendelian factor") allowed breeders to exploit family relationships and more accurately estimate breeding values (Fisher 1919; Wright 1921). Nevertheless, this concept was still a theoretical construct and assumed an infinitesimal model for complex traits: it could not account for deviations from expected genetic relationships due to finite numbers of causal loci and differential effects across loci. Breeding 3 addressed these limitations through marker technology and linear regression models. In Breeding 3, genetic markers represent causal loci as QTL, allowing their contribution to phenotypic variability to be depicted explicitly. However, these innovations introduced both technological and statistical challenges. Due to ascertainment bias (sampling bias of markers during their development), genetic markers in Breeding 3 generally do not assay the whole pool of DNA polymorphisms in any given population (Heslot et al. 2013). Furthermore, utilizing marker information typically implies estimating effects on many more loci ( $p$ genotypic variables) than there are observations at the genotype level ( $n$ plant genotypes, i.e., instances with distinct genetics): $n \ll p$. As a result, model parameters cannot be solved without simplifying assumptions. So, even though Breeding 3 models may accurately predict genetic merit, they should not be used to infer effects of many QTL. This curse of dimensionality is a critical issue in Breeding 3, and it severely hampers applications based on estimated QTL effects, especially for complex traits. In models based on few markers, omitted marker information results in models that fail to account for the confounding effects of loci. For example, apparent QTL effects may be caused by unobserved causal loci that are physically linked to genetic markers. In marker screens involving single-marker analyses or stepwise model selections (e.g., association mapping analyses), estimated effects may fluctuate randomly around their true values. These random fluctuations can reach such extremes that the most significant estimates might be highly overestimated in magnitude: this is the winner's curse, also known as the Beavis effect in plant breeding (Beavis 1998; Xu 2003). Finally, when $n \ll p$, models fitting all marker effects simultaneously must make assumptions about their distribution (typically, a normal distribution around zero) by means of regularization, such that their estimates are distorted (Gianola 2013). All three aforementioned cases illustrate the common issue of estimation bias regarding QTL effects in an $n \ll p$ context, and therefore stress the inadequacy of observed marker associations to make causal inferences about complex traits in Breeding 3 (Fig. 2).

The defining goal of Breeding 4 is to alleviate the issues of ascertainment bias and high dimensionality $(n \ll p)$ in order to make reliable inferences about numerous effects of loci. More exhaustive assays of genetic variability, using whole-genome or haplotype sequence data, will allow scientists to directly query the effects of observed loci, instead of estimating them indirectly through linked genetic markers. Furthermore, analyses involving higher $n$ and/or lower $p$ will permit more reliable inference of causal loci. This new framework will make use of sequence or image data and will therefore benefit from innovative machine learning approaches, different from the linear regression models typically used in Breeding 3 (e.g., see Ben-Hur et al. 2008 and
Fig. 2 The shift in statistical framework between Breeding 3 and Breeding 4. Breeding 4 will aim to avoid the curse of dimensionality $(n \ll p)$ which precludes the inference of causal loci, especially for traits controlled by many causal loci. Machine learning techniques such as neural networks will be useful under this novel framework, to model effects of DNA polymorphisms on endophenotypes and predict component traits from high-throughput phenotyping (HTP) data

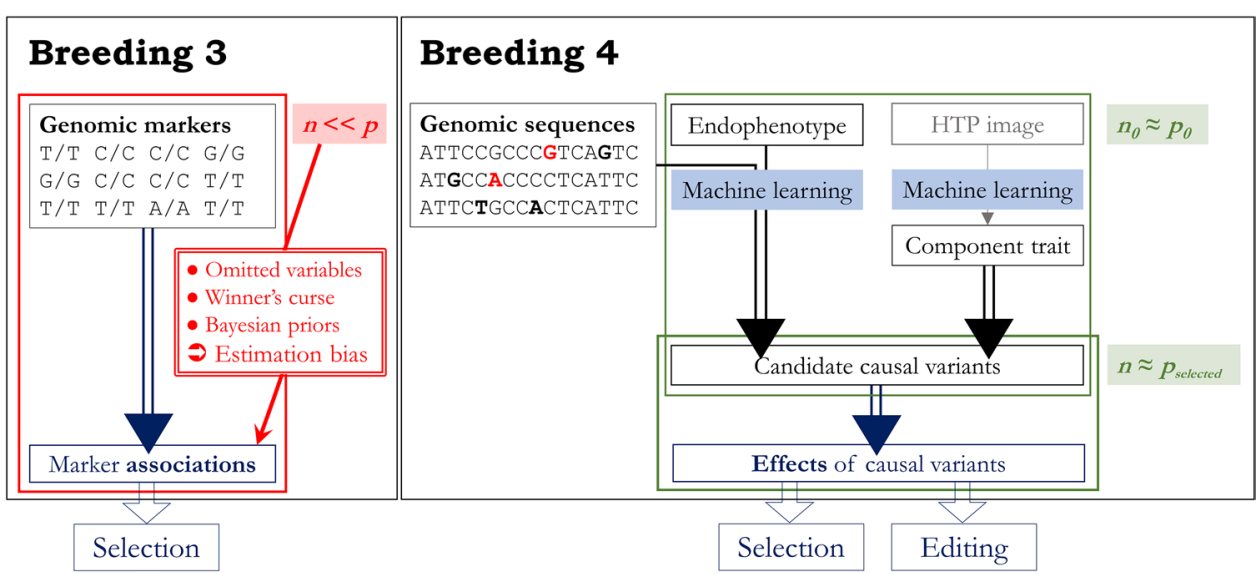


Angermueller et al. 2016 for general introductions to such techniques in computational biology). Inference of causal loci will be key to define plausible plant ideotypes for guiding biological designs of improved varieties.

\section{The statistical and technological relevance of Breeding 4}

While Breeding 3 used marker associations to make selections, Breeding 4 will efficiently integrate genotype and phenotype data to identify causal genetic features for breeders to make selections and perform biological interventions based on genetic transformation technologies (Wallace et al. 2018). Already such technologies have been used to transfer individual genes into plant varieties or to make precise edits at key genes controlling high-value traits (Breeding 4.1). For example, transgenic modification has been used to introduce insect resistance traits into maize cultivars (Koziel et al. 1993) or reduce browning in apple (Armstrong and Lane 2013), and gene editing by clustered regularly interspaced short palindromic repeats (CRISPR) has already produced useful characteristics by modifying single genes, like ARGOS8 for increased drought tolerance in maize (Shi et al. 2017). Continued research in gene editing is paving the way to parallel editing across the genome, as was demonstrated by methods for simultaneous gene editing of 16,006 sites in yeast samples (Sharon et al. 2018). Future iterations of the CRISPR technology will likely make it possible to modify complex traits in plants with thousands of directed edits (Breeding 4.2). Improvements in genetic transformation techniques will further facilitate gene transfer and gene editing, particularly in grass species (Lowe et al. 2016), making parallel gene editing all the more practical as a crop improvement tool. Compared to marker-assisted breeding, editing will create exciting new possibilities. It will accelerate the breeding cycle and reduce reliance on random recombination events for genetic improvement. It will also allow breeders to shorten the breeding process because, contrary to multi-parental programs in Breeding 3, combining the best polymorphisms from many plant genotypes will not require more crosses. Gene editing will allow the development of cultivars with fewer deleterious mutations, both in low-recombination regions where genetic burden has accumulated due to low crossover rate and genetic hitchhiking, and in high-recombination regions where they are difficult to purge via recombination due to their close proximity to beneficial alleles (Rodgers-Melnick et al. 2015; Ramu et al. 2017).

QTL models in Breeding 3 commonly rely on linear relationships between genetic markers and agronomic traits. Linear mixed models and related frameworks have been widely used to predict agronomic traits in genomic prediction (Meuwissen et al. 2001) and identify QTL in association mapping (Yu et al. 2006). These models have successfully guided breeding decisions; however, the next phase of plant breeding, Breeding 4, will aim to estimate locus effects accurately, not only to predict breeding values but also to infer causal loci (Fig. 2). Therefore, Breeding 4 will have to either reduce $p$ or increase $n$. High-throughput phenotyping on simpler component traits (morphological and physiological traits) can be used to produce data on more genotypes, or endophenotypes (intermediate molecular traits such as gene expression levels or metabolic activity) can be used to provide many phenotypes per genotype (e.g., using expression data on every gene in a genotype, as in Kremling et al. 2018). Alternatively, the number of loci assayed can be decreased by focusing only on single genes or regions of the genome (e.g., focusing on polymorphisms in promoter regions and the UTR, as in Washburn et al. 2018). Relationships between polymorphisms and phenotypes can be depicted more accurately with genetically simpler traits than with the agronomic traits used in Breeding 3. In Breeding 4, using simpler endophenotypes or component traits as phenotypes should therefore reduce the required model complexity. Biologically meaningful models are critical, because Breeding 4 will require a significantly better understanding of the relationship between genotypes and phenotypes (the genotype-phenotype map) to define genetic ideotypes consisting of favorable alleles at causal loci for agronomic traits. This goal differs substantially from those in Breeding 3, where genetic data are used to mark loci rather than interrogate them directly, and will involve data types and modeling approaches that are complementary to those used in Breeding 3. Breeding 4 will use more functionally relevant features to infer causal loci (e.g., DNA sequence data rather than genetic markers) and will involve different models than simple linear regressions. Sophisticated pattern recognition algorithms will be critical in Breeding 4. Complex machine learning models will be needed to interrogate sequence data and capture nonlinear effects of polymorphisms on endophenotypes, or to estimate component traits based on high-throughput phenotyping image data (Fig. 2). These include nonlinear models such as neural networks, consisting of latent variables arranged in hidden layers (Bishop 1995). In Breeding 4, neural networks such as convolutional and recurrent neural networks $(\mathrm{CNNs}$ and RNNs, respectively) may be particularly appropriate, not only because they can capture nonlinear relationships to their output but also because they are designed to use sequence or image data as inputs. These models have been developed since the 1980s (LeCun et al. 1998; Deng et al. 2013), but their widespread applications to biological problems emerged much later, around 2014 (Angermueller et al. 2016), thanks to progress in computer hardware technology (LeCun et al. 2015) and critical improvements in 
optimization techniques for neural networks (e.g., Kingma and Ba 2014; Ioffe and Szegedy 2015).

\section{Using machine learning to prioritize causal loci in Breeding 4}

\section{Detecting causal polymorphisms from sequence data}

Starting from Sanger sequencing in the 1970s, sequencing technologies have progressed toward higher throughput and lower cost per nucleotide (Metzker 2005). The current state of sequencing technologies consists of short reads from next-generation sequencing, used together with less accurate but considerably longer reads from Pacific Biosciences or Oxford Nanopore Technologies sequencers (Goodwin et al. 2016). Improvements in sequencing technologies will make it easier to build and update reference genomes and assemblies, which will be useful for cataloging causal variants within their haplotype context in breeding populations. Haplotypes are small portions of chromosomes that are the basic units of heredity. They consist of DNA sequences which can be inferred based on marker data or low-depth, highly multiplexed sequencing data. Haplotype graphs are convenient computational frameworks to accurately infer haplotype sequences, as was made evident from studies in humans (Eggertsson et al. 2017). These graphs will provide a concise representation of genetic diversity from inexpensive genetic data, which should make Breeding 3 techniques (especially genomic prediction) accessible to breeders with low computer memory resources and limited genotyping budgets. In Breeding 4, haplotype graphs will provide fully contextualized DNA sequence information to genetic models. Such inputs should be more relevant depictions of the genetic bases of traits than genetic markers and should assay polymorphisms more thoroughly, hence remedying the issue of ascertainment bias. Furthermore, they will provide contextual information about possible effects of loci, e.g., a single nucleotide polymorphism (SNP) acting by disrupting a $c i s$-regulatory motif. Finally, DNA sequences may be augmented with functional annotations at the level of single nucleotides such that input sequences include external information about their potential effects. Functional annotations may include characteristics about chromatin accessibility, which has been shown to account for a substantial amount of phenotypic variability in maize (Rodgers-Melnick et al. 2016), or information about selection pressure. For example, genomic evolutionary rate profiling (GERP) scores (Davydov et al. 2010) have been shown to correlate with SNP effects in maize (Yang et al. 2017). In general, haplotypes will contain detailed knowledge about nucleotides, genes and sub-chromosomal regions in a species, and should help extend genomic analyses to cross-species research, allowing breeders to use DNA information across species boundaries (Mace et al. 2013).

Breeding 1 and Breeding 2 relied on macroscopic traits to select the best individuals from a population. Breeding 3 has begun to use endophenotypes. Recent studies have combined transcriptome- and genome-wide association studies to increase power to detect causal genes (Kremling et al. 2018) and used metabolomic data to predict hybrid performance in maize (Schrag et al. 2018) and rice (Xu et al. 2016). Breeding 4 will extend the use of endophenotypes to precisely relate DNA information to endophenotypes and whole-plant performance. While suitable for analyzing additive effects of polymorphisms, typical Breeding 3 models are not equipped to reflect the effects of polymorphisms on endophenotypes in the context of particular DNA motifs. For example, they cannot accommodate differences in genomic position of functionally relevant patterns nor account for motifs occurring at multiple locations in a given genome. Moreover, these models cannot exploit biological replication within genotypes. While effects governing endophenotypes are likely similar, especially within a family of genes or regulatory regions, Breeding 3 models usually do not allow estimated effects to be shared across these similar contexts. Conversely, machine learning models have been designed to accommodate variability in occurrence of motifs and similarity in their effect across genomic regions. These include models based on counts or vector representations of $k$-mers in regulatory regions (Mejia-Guerra and Buckler 2017), as well as neural networks such as CNNs and RNNs which can recognize motifs occurring anywhere within sequences (by local scans in convolutions, or explicit dependencies in recurrences). Thus, machine learning can alleviate the limitations of Breeding 3 models and incorporate relevant information beyond genetic marker effects. Recent examples of machine learning applied to biological sequences include CNNs that use promoter sequences, coded as vectors of four binary variables (indicating each of the possible DNA nucleotides), to predict endophenotypes such as epigenetic marks (Angermueller et al. 2017) or transcription levels (Washburn et al. 2018; Zhou et al. 2018). Neural networks in these applications not only increased accuracy on prediction tasks involving DNA sequences, but also offered the possibility to prioritize variants based on their effects in the model, either by in silico mutagenesis (Zhou and Troyanskaya 2015) or by gradient computations (Washburn et al. 2018). Such prioritization based on endophenotypes holds great potential to enrich pools of polymorphisms for causal variants, as was recently suggested in humans (Zhou et al. 2018).

Another shortcoming of typical Breeding 3 models is their inability to easily accommodate equifinality issues: in QTL models, individual markers usually cannot represent the effect of multiple mutations causing the same 
phenotypes (e.g., different polymorphisms producing different STOP codons, all causing the coded protein to be non-functional). Differently, neural networks may capture biologically relevant effects of polymorphisms beyond additive effects and should be able to model effects of interactions between polymorphisms in local nonlinear functions (Poggio et al. 2017). In particular, locally connected neural networks (which include CNNs) may capture local epistatic effects within haplotypes, which are likely to be biologically meaningful (especially in genic regions) and inherited together (therefore contributing to additive genetic variance within a small breeding population). Therefore, machine learning analyses, complementary to Breeding 3, could increase power to detect causal variants of agronomic interest, because of important advantages: (1) they have the ability to use fully contextualized sequences as input data; (2) they can infer nonlinear relationships between polymorphisms and endophenotypes; and (3) they might be used in a $n>p$ context, where inference about genetic effects might be possible (Fig. 2).

\section{Inferring morphological and physiological traits from image data}

Most agronomic traits are controlled by complex physiological processes. Breaking complex traits into multiple phenotypic measurements may allow breeders to target different aspects of the trait, which is useful when breeding for consistency across environments (e.g., selecting for drought adaptation instead of directly for grain yield, which may depend on different adaptive traits depending on breeding environments; Cooper et al. 2009). Trait decomposition is all the more useful in marker-assisted breeding: splitting complex phenotypes into simpler component phenotypes also means splitting a highly quantitative trait into multiple traits with a simpler genetic basis (Hammer et al. 2005; Messina et al. 2011). However, direct assays of physiological traits (e.g., measurements of stomatal conductance or wateruse efficiency) have critical disadvantages which preclude their utilization in breeding: (1) they are typically expensive and time-consuming; (2) they often must be performed in controlled conditions; and (3) they can be destructive. Such limitations can be overcome by imaging technologies which allow for high-throughput phenotyping by either rapid measurements of individual samples by field robots (Andrade-Sanchez et al. 2014), or single measurements of many samples at once by unmanned aerial vehicles (UAVs; Shi et al. 2016). Imaging technologies may capture radiation at different spectral ranges (visible range, near-infrared, farinfrared, etc.) with discrete or quasi-continuous wavelength resolutions, or other signals such as reflected laser pulses (LiDAR) which can be used to construct 3D images (Araus and Cairns 2014). Physiological and morphological traits can then be derived from images manually, e.g., normalized difference vegetation index (NDVI) calculated from reflectance at specific wavelengths (Sims and Gamon 2002), or photosynthetic rate calculated from chlorophyll fluorescence (Meyer and Genty 1998). Such derivation can also be automatic, however, rather than defined a priori and explicitly, and may thereby benefit from recent innovative machine learning approaches.

Traditionally, extracting trait information from image data on a single instance (e.g., an individual plant) has consisted of two steps: (1) segmentation, in which regions of interest for feature extraction are isolated (e.g., leaf area out of the background); and (2) analysis of structure, in which component traits are predicted from regions of interest (e.g., leaf counts from leaf area) (Spalding and Miller 2013). With the advent of neural networks, this framework may shift to a single processing step in which component traits are predicted directly from raw images, thereby moving away from trait-specific handcrafted predictors toward automatic abstract representations for predicting morphological and/ or physiological traits (Singh et al. 2018). Examples of such approaches include CNN-like models for inferring leaf count from images of Arabidopsis plants (Giuffrida et al. 2018) or for classifying disease from leaf images in various plant species (Mohanty et al. 2016). Notably, DeChant et al. (2017) have shown the accuracy of CNNs for predicting northern leaf blight occurrence in maize from UAV images, demonstrating the applicability of neural networks for highthroughput phenotyping in the field.

While neural networks, especially CNNs, are promising for automatically predicting component traits from image data on a single instance, segmenting multiple instances from field images remains a challenge. As part of this effort, instance segmentation may be performed manually (Tsaftaris et al. 2016) or automatically, the latter being based on GPS coordinates (e.g., delineating plots in field images from UAVs; Shi et al. 2016) or pattern recognition. In particular, region-based CNNs have been used to simultaneously classify and isolate instances in global images (Girshick et al. 2014; He et al. 2017). One example comes from Jin et al. (2018) who used region-based CNNs to detect maize stems from field images.

Despite the advantages of automatic pattern recognition for image analysis, strategies based on neural networks for high-throughput phenotyping will face the challenge of interpretability inherent to the "black-box" nature of this type of models: predictions may depend on confounded factors rather than meaningful physiological or morphological characteristics. However, useful techniques exist to train models to be more robust to confounders, such as size or orientation of patterns in the image, e.g., data augmentation with shifted, re-scaled and/or rotated images (Bishop 2006). Hence, the combinations of machine learning models 
such as CNNs, and practices for robust training such as data augmentation should ensure reliable inference of component traits from high-throughput phenotyping images.

For each genotype, estimation of component traits would provide useful replication over time, as opposed to replication across plants. This type of replication should be useful to increase the prediction accuracy for component traits on each plant, making use of correlation of measurements over time to fit time-series functions to the data (e.g., logistic regression of plant height over time) for estimating component traits as parameters of such functions (e.g., a slope parameter reflecting growth rate) (van Eeuwijk et al. 2018). Nondestructive and accurate measurements of component traits by analysis of high-throughput phenotyping images, relying on useful machine learning approaches and possibly time-series analyses, should therefore allow breeders to test more plant genotypes in the field (higher $n$ ), with high enough accuracy for applications in Breeding 4.

With high enough $n$, quantitative genetics models may be used to predict component traits and detect genetic markers linked to their causal variants (Messina et al. 2011). In Breeding 3, component traits predicted from DNA information could be incorporated in genomic prediction models, thereby increasing their predictive ability for agronomic traits. Examples of this strategy are prediction analyses based on multivariate linear mixed models (Sun et al. 2017) or nonlinear crop growth models (Messina et al. 2018). In Breeding 4, markers or genomic regions (e.g., haplotypes) showing significant associations with component traits could be used to prioritize variants. In all likelihood, this strategy, similar to the preselection of variants based on endophenotypes, could effectively alleviate the $n \ll p$ issue for subsequent analyses on agronomic traits (Fig. 2).

\section{Moving from $n \ll p$ to $n>p$ for testing variants and developing improved varieties}

Ultimately, Breeding 4 will aim to detect causal variants as precisely as possible for subsequent breeding or editing, using approaches complementary to traditional QTL mapping techniques. This effort will rely on various types of data: DNA information (either in the form of genetic markers or DNA sequences), genomic annotation (on epigenetic status or evolutionary constraints) and phenotypic data, including both agronomic traits of interest and intermediate traits such as endophenotypes or component traits. Breeding 4 has become possible because of rapid progress in genotyping technologies (DNA sequencing, measurement of endophenotypes and inference of haplotypes) as well as phenotyping technologies (image acquisition and robotics). This phase will be characterized by a shift in focus from genetic marker data to well-annotated haplotype data. The shift from genetic markers to haplotypes will further reduce the cost of genomic prediction and help expand Breeding 3 to new crops and programs. In Breeding 4, it will allow contextualized sequence data to be used as inputs to models predicting endophenotypes. Fortuitously, Breeding 4 is concurrent with the development of machine learning methodologies, in particular neural networks such as CNNs or RNNs, which are appropriate for estimating the effects of polymorphisms on endophenotypes (from DNA sequences) or analyzing high-throughput phenotyping data for predicting component traits (from images). Realistic depiction of the genotype-phenotype map for these simpler traits will allow to estimate effects for prioritizing a handful of putative causal variants that are worth assessing in subsequent analyses on agronomic traits (Fig. 2). Two plant genotypes (e.g., inbred lines in maize) may differ from one another by tens of millions of genomic locations. This prioritization process will dramatically reduce the pool of variants to be tested, using either quantitative analyses (e.g., Breeding 3 methods) or transformation technologies (e.g., CRISPR gene editing). Following prioritization, a testing phase will define plant ideotypes according to which improved varieties will be developed, by selection or biological design. In maize, we envision the process of nominating thousands of edits, then editing nominated loci simultaneously in a homogeneous inbred background, to reduce the $n \ll p$ issue (with only thousands of loci segregating) and to eliminate confounding by physical linkage of loci. Then, with reasonable scale in field trials, effects of loci can be measured to define reliable plant ideotypes for developing improved varieties by genomic selection or genome editing.

Author contribution statement GPR, SEJ and ESB all contributed to developing the article's outline, reviewing the literature and writing the manuscript.

Acknowledgements We would like to thank two anonymous reviewers for their helpful comments about the manuscript. This work was funded by the USDA-ARS, the National Science Foundation Plant Genome Research Project no. 1238014, ARPA-E (Advanced Research Projects Agency-Energy) within the US Department of Energy, and the support of the American People provided to the Feed the Future Innovation Lab for Collaborative Research on Sorghum and Millet through the US Agency for International Development (USAID). The contents are the sole responsibility of the authors and do not necessarily reflect the views of the USDA, USAID or the US Government. Program activities are funded by the US Agency for International Development (USAID) under Cooperative Agreement No. AID-OAA-A-13-00047.

\section{Compliance with ethical standards}

Conflict of interest On behalf of all authors, the corresponding author states that there is no conflict of interest.

Open Access This article is distributed under the terms of the Creative Commons Attribution 4.0 International License (http://creativeco 
mmons.org/licenses/by/4.0/), which permits unrestricted use, distribution, and reproduction in any medium, provided you give appropriate credit to the original author(s) and the source, provide a link to the Creative Commons license, and indicate if changes were made.

\section{References}

Andrade-Sanchez P, Gore MA, Heun JT et al (2014) Development and evaluation of a field-based high-throughput phenotyping platform. Funct Plant Biol 41:68-79

Angermueller C, Pärnamaa T, Parts L, Stegle O (2016) Deep learning for computational biology. Mol Syst Biol 12:878

Angermueller C, Lee HJ, Reik W, Stegle O (2017) DeepCpG: accurate prediction of single-cell DNA methylation states using deep learning. Genome Biol 18:67

Araus JL, Cairns JE (2014) Field high-throughput phenotyping: the new crop breeding frontier. Trends Plant Sci 19:52-61

Armstrong J, Lane WD (2013) Genetically modified reduced-browning fruit-producing plant and produced fruit thereof, and method of obtaining such. US Patent

Beavis WD (1998) QTL analyses: power, precision, and accuracy. Mol Dissection Complex Traits 1998:145-162

Ben-Hur A, Ong CS, Sonnenburg S et al (2008) Support vector machines and kernels for computational biology. PLoS Comput Biol 4:e1000173

Bernardo R (2002) Breeding for quantitative traits in plants. Stemma Press, Woodbury

Bevan MW, Uauy C, Wulff BBH et al (2017) Genomic innovation for crop improvement. Nature 543:346-354

Biffen RH (1905) Mendel's laws of inheritance and wheat breeding. J Agric Sci 1:4-48

Bishop CM (2006) Pattern recognition and machine learning (information science and statistics). Springer, Berlin

Bishop CM, of Neural Computing Christopher (1995) Neural networks for pattern recognition. Clarendon Press, Oxford

Briggs WH, McMullen MD, Gaut BS, Doebley J (2007) Linkage mapping of domestication loci in a large maize teosinte backcross resource. Genetics 177:1915-1928

Cochran WG, Cox GM (1950) Experimental designs. Wiley, Oxford

Cooper M, van Eeuwijk FA, Hammer GL et al (2009) Modeling QTL for complex traits: detection and context for plant breeding. Curr Opin Plant Biol 12:231-240

Correns CE (1900) Mendel's Regel uber das Verhalten der Nachkommenschaft der Rassenbastarde. Ber Dtsch Bot Ges 18:158-167

Davydov EV, Goode DL, Sirota M et al (2010) Identifying a high fraction of the human genome to be under selective constraint using GERP ++. PLoS Comput Biol 6:e1001025

De Vries H (1900) Sur la loi de disjonction des hybrides. CR Acad Sci 130:845-847

DeChant C, Wiesner-Hanks T, Chen S et al (2017) Automated identification of northern leaf blight-infected maize plants from field imagery using deep learning. Phytopathology 107:1426-1432

Deng L, Hinton G, Kingsbury B (2013) New types of deep neural network learning for speech recognition and related applications: an overview. In: 2013 IEEE international conference on acoustics, speech and signal processing. ieeexplore.ieee.org, pp 8599-8603

Doebley J, Stec A, Wendel J, Edwards M (1990) Genetic and morphological analysis of a maize-teosinte F2 population: implications for the origin of maize. Proc Natl Acad Sci U S A 87:9888-9892
Eggertsson HP, Jonsson H, Kristmundsdottir S et al (2017) Graphtyper enables population-scale genotyping using pangenome graphs. Nat Genet 49:1654-1660

Fisher RA (1919) The correlation between relatives on the supposition of mendelian inheritance. Earth Environ Sci Trans R Soc Edinb 52:399-433

Fisher RA (1930) The genetical theory of natural selection. Clarendon Press, Oxford

Fisher RA (1935) The design of experiments. Oliver and Boyd, Edinburgh

Flor HH (1971) Current status of the gene-for-gene concept. Annu Rev Phytopathol 9:275-296

Gianola D (2013) Priors in whole-genome regression: the bayesian alphabet returns. Genetics 194:573-596

Girshick R, Donahue J, Darrell T, Malik J (2014) Rich feature hierarchies for accurate object detection and semantic segmentation. In: Proceedings of the IEEE conference on computer vision and pattern recognition, pp 580-587

Giuffrida MV, Doerner P, Tsaftaris SA (2018) Pheno-deep counter: a unified and versatile deep learning architecture for leaf counting. Plant J 96:880-890

Goodwin S, McPherson JD, McCombie WR (2016) Coming of age: ten years of next-generation sequencing technologies. Nat Rev Genet 17:333-351

Hammer GL, Chapman S, van Oosterom E, Podlich DW (2005) Trait physiology and crop modelling as a framework to link phenotypic complexity to underlying genetic systems. Aust J Agric Res 56:947-960

He K, Gkioxari G, Dollár P, Girshick R (2017) Mask R-CNN. In: 2017 IEEE international conference on computer vision (ICCV), pp $2980-2988$

Hedden P (2003) The genes of the green revolution. Trends Genet 19:5-9

Henderson CR, Kempthorne O, Searle SR, von Krosigk CM (1959) The estimation of environmental and genetic trends from records subject to culling. Biometrics 15:192-218

Heslot N, Rutkoski J, Poland J et al (2013) Impact of marker ascertainment bias on genomic selection accuracy and estimates of genetic diversity. PLoS ONE 8:e74612

Heslot N, Jannink J-L, Sorrells ME (2015) Perspectives for genomic selection applications and research in plants. Crop Sci 55:1-12

Hospital F, Chevalet C, Mulsant P (1992) Using markers in gene introgression breeding programs. Genetics 132:1199-1210

Huang X, Han B (2014) Natural variations and genome-wide association studies in crop plants. Annu Rev Plant Biol 65:531-551

Ioffe S, Szegedy C (2015) Batch normalization: accelerating deep network training by reducing internal covariate shift. arXiv [cs.LG]

Jin S, Su Y, Gao S et al (2018) Deep learning: individual maize segmentation from terrestrial lidar data using faster R-CNN and regional growth algorithms. Front Plant Sci 9:866

Kingma DP, Ba J (2014) Adam: a method for stochastic optimization. arXiv [cs.LG]

Kovach MJ, Sweeney MT, McCouch SR (2007) New insights into the history of rice domestication. Trends Genet 23:578-587

Koziel MG, Beland GL, Bowman C et al (1993) Field performance of elite transgenic maize plants expressing an insecticidal protein derived from Bacillus thuringiensis. Biotechnology 11:194

Kremling KAG, Chen S-Y, Su M-H et al (2018) Dysregulation of expression correlates with rare-allele burden and fitness loss in maize. Nature 555:520-523

Lecun Y, Bottou L, Bengio Y, Haffner P (1998) Gradient-based learning applied to document recognition. Proc IEEE 86:2278-2324

LeCun Y, Bengio Y, Hinton G (2015) Deep learning. Nature 521:436-444 
Lowe K, Wu E, Wang $\mathrm{N}$ et al (2016) Morphogenic regulators baby boom and wuschel improve monocot transformation. Plant Cell 28:1998-2015

Lush JL (1937) Animal breeding plans. Iowa State College Press, Ames

Mace ES, Hunt CH, Jordan DR (2013) Supermodels: sorghum and maize provide mutual insight into the genetics of flowering time. Theor Appl Genet 126:1377-1395

Mejia-Guerra MK, Buckler ES (2017) k-mer grammar uncovers maize regulatory architecture. bioRxiv 222927

Mendel G (1866) Versuche über Pflanzenhybriden. Verh des naturforschenden Ver in Brunn 4(3):44

Messina CD, Podlich D, Dong Z et al (2011) Yield-trait performance landscapes: from theory to application in breeding maize for drought tolerance. J Exp Bot 62:855-868

Messina CD, Technow F, Tang T et al (2018) Leveraging biological insight and environmental variation to improve phenotypic prediction: integrating crop growth models (CGM) with whole genome prediction (WGP). Eur J Agron. https://doi.org/10.1016/j. eja.2018.01.007

Metzker ML (2005) Emerging technologies in DNA sequencing. Genome Res 15:1767-1776

Meuwissen TH, Hayes BJ, Goddard ME (2001) Prediction of total genetic value using genome-wide dense marker maps. Genetics 157:1819-1829

Meyer S, Genty B (1998) Mapping intercellular CO2 mole fraction (C i) in Rosa rubiginosa leaves fed with abscisic acid by using chlorophyll fluorescence imaging: significance of $\mathrm{C}$ i estimated from leaf gas exchange. Plant Physiol 116:947-957

Mohanty SP, Hughes DP, Salathé M (2016) Using deep learning for image-based plant disease detection. Front Plant Sci 7:1419

Orr HA (1998) The population genetics of adaptation: the distribution of factors fixed during adaptive evolution. Evolution 52:935-949

Orr HA (2005) The genetic theory of adaptation: a brief history. Nat Rev Genet 6:119-127

Panter DM, Allen FL (1995) Using best linear unbiased predictions to enhance breeding for yield in soybean: I. Choosing parents. Crop Sci 35:397-405

Poggio T, Mhaskar H, Rosasco L et al (2017) Why and when can deep-but not shallow-networks avoid the curse of dimensionality: a review. Int J Autom Comput 14:503-519

Ramu P, Esuma W, Kawuki R et al (2017) Cassava haplotype map highlights fixation of deleterious mutations during clonal propagation. Nat Genet 49:959-963

Rodgers-Melnick E, Bradbury PJ, Elshire RJ et al (2015) Recombination in diverse maize is stable, predictable, and associated with genetic load. Proc Natl Acad Sci U S A 112:3823-3828

Rodgers-Melnick E, Vera DL, Bass HW, Buckler ES (2016) Open chromatin reveals the functional maize genome. Proc Natl Acad Sci U S A 113:E3177-E3184

Schrag TA, Westhues M, Schipprack W et al (2018) Beyond genomic prediction: combining different types of omics data can improve prediction of hybrid performance in maize. Genetics 208:1373-1385

Sharon E, Chen S-AA, Khosla NM et al (2018) Functional genetic variants revealed by massively parallel precise genome editing. Cell 175:544-557.e16

Shi Y, Thomasson JA, Murray SC et al (2016) Unmanned aerial vehicles for high-throughput phenotyping and agronomic research. PLoS ONE 11:e159781

Shi J, Gao H, Wang H et al (2017) ARGOS 8 variants generated by CRISPR-Cas9 improve maize grain yield under field drought stress conditions. Plant Biotechnol J 15:207-216
Sims DA, Gamon JA (2002) Relationships between leaf pigment content and spectral reflectance across a wide range of species, leaf structures and developmental stages. Remote Sens Environ $81: 337-354$

Singh AK, Ganapathysubramanian B, Sarkar S, Singh A (2018) Deep learning for plant stress phenotyping: trends and future perspectives. Trends Plant Sci 23:883-898

Soller M, Brody T, Genizi A (1976) On the power of experimental designs for the detection of linkage between marker loci and quantitative loci in crosses between inbred lines. Theor Appl Genet 47:35-39

Spalding EP, Miller ND (2013) Image analysis is driving a renaissance in growth measurement. Curr Opin Plant Biol 16:100-104

Stitzer MC, Ross-Ibarra J (2018) Maize domestication and gene interaction. New Phytol 220:395-408

Stuber CW, Moll RH, Goodman MM et al (1980) Allozyme frequency changes associated with selection for increased grain yield in maize (Zea mays L.). Genetics 95:225-236

Sun J, Rutkoski JE, Poland JA et al (2017) Multitrait, random regression, or simple repeatability model in high-throughput phenotyping data improve genomic prediction for wheat grain yield. Plant Genome 10.: https://doi.org/10.3835/plantgenome2016.11.0111

Tanksley SD, Medina-Filho H, Rick CM (1982) Use of naturally-occurring enzyme variation to detect and map genes controlling quantitative traits in an interspecific backcross of tomato. Heredity 49:11

Tsaftaris SA, Minervini M, Scharr H (2016) Machine learning for plant phenotyping needs image processing. Trends Plant Sci 21:989-991

Turelli M (2017) Commentary: Fisher's infinitesimal model: a story for the ages. Theor Popul Biol 118:46-49

van Eeuwijk FA, Bustos-Korts D, Millet EJ et al (2018) Modelling strategies for assessing and increasing the effectiveness of new phenotyping techniques in plant breeding. Plant Sci. https://doi. org/10.1016/j.plantsci.2018.06.018

Wallace JG, Rodgers-Melnick E, Buckler ES (2018) On the road to Breeding 4.0: unraveling the good, the bad, and the boring of crop quantitative genomics. Annu Rev Genet 52:421-444

Washburn JD, Guerra MKM, Ramstein G et al (2018) Evolutionarily informed deep learning methods: Predicting transcript abundance from DNA sequence. bioRxiv 372367

Wright S (1921) Systems of mating. Genetics 6:111-178

$\mathrm{Xu} \mathrm{S} \mathrm{(2003)} \mathrm{Theoretical} \mathrm{basis} \mathrm{of} \mathrm{the} \mathrm{Beavis} \mathrm{effect.} \mathrm{Genetics}$ 165:2259-2268

Xu S, Xu Y, Gong L, Zhang Q (2016) Metabolomic prediction of yield in hybrid rice. Plant J 88:219-227

Yang J, Mezmouk S, Baumgarten A et al (2017) Incomplete dominance of deleterious alleles contributes substantially to trait variation and heterosis in maize. PLoS Genet 13:e1007019

Yu J, Pressoir G, Briggs WH et al (2006) A unified mixed-model method for association mapping that accounts for multiple levels of relatedness. Nat Genet 38:203-208

Zhou J, Troyanskaya OG (2015) Predicting effects of noncoding variants with deep learning-based sequence model. Nat Methods 12:931-934

Zhou J, Theesfeld CL, Yao K et al (2018) Deep learning sequencebased $a b$ initio prediction of variant effects on expression and disease risk. Nat Genet 50:1171-1179 